13

\title{
Влияние адсорбции на работу выхода и проводимость углеродных наноструктур: противоречивость экспериментальных данных
}

\author{
(C) С.Ю. Давыдов, ${ }^{1,2}$ O.В. Посредник ${ }^{3}$ \\ ${ }^{1}$ Физико-технический институт им. А.Ф. Иофрфе РАН, \\ 194021 Санкт-Петербург, Россия \\ ${ }^{2}$ Университет ИТМО, \\ 197101 Санкт-Петербург, Россия \\ ${ }^{3}$ Санкт-Петербургский государственный электротехнический университет (ЛЭтИ), \\ 197376 Санкт-Петербург, Россия \\ e-mail: Sergei_Davydov@mail.ru
}

(Поступило в Редакцию 4 марта 2016 г. В окончательной редакции 12 июля 2016 г.)

В рамках разработанной ранее одним из авторов теории, критически проанализированы имеющиеся экспериментальные работы по влиянию молекул газа на работу выхода и проводимость слоев наноструктурированных графита и графена. Продемонстрировано, что эти работы содержат ряд противоречивых результатов. Обсуждена применимость предложенной теории к углеродным наноструктурам.

DOI: $10.21883 /$ JTF.2017.04.44330.1790

\section{Введение}

Рассматривая явление адсорбции, можно выделить два главных эффекта [1]. Первый эффект - изменение работы выхода адсорбционной системы $\Delta \phi$, обусловленное обменом электронами между адатомом и подложкой (переход заряда). Такой обмен приводит к появлению у адатома отличного от нуля заряда $Z_{a}$, способствующего $\left(Z_{a}>0\right)$ или препятствующего $\left(Z_{a}<0\right)$ выходу электронов из субстрата и, следовательно, понижающего $(\Delta \phi<0)$ или повышающего $(\Delta \phi>0)$ работу выхода системы. В первом случае происходит переход электронов с адатома-донора в подложку, во втором из подложки на адатом-акцептор. Если до адсорбции атома его одноэлектронное состояние, работающее на переход заряда, было заполнено, а после адсорбции число заполнения этого состояния стало равным $n_{a}$, то $Z_{a}=1-n_{a}$. Следовательно, $Z_{a}$-электронов уходят в зону проводимости субстрата. Если это одноэлектронное состояние изначально было пустым (содержало одну дырку), а в результате адсорбции приобрело $n_{a}$-электронов, то заряд адатома принимает значение $Z_{a}=-n_{a}$, а в валентной зоне субстрата возникает $n_{a}$-дырок. Если же характеризовать этот атом двуэлектронной орбиталью, то $Z_{a}=1-n_{a}$. Подчеркнем, что во всех случаях величина $\left|Z_{a}\right|$ равна переходу заряда.

Второй эффект, вызываемый адсорбцией, это изменение поверхностной проводимости подложки $\Delta G[1]$. Причины этого эффекта двояки. Во-первых, изменяется поверхностная концентрация носителей $\Delta n$ : адатомыдоноры (-акцепторы) увеличивают (уменьшают) проводимость подложки $n$-типа и, наоборот, уменьшают (увеличивают) проводимость подложки р-типа. Во-вторых, адчастицы представляют собой дополнительные центры рассеяния, что, вообще говоря, должно влиять на поверхностную подвижность носителей $\mu$.
Начало систематических исследований одновременных изменений поверхностной проводимости и работы выхода положили экспериментальные [2,3] и теоретические [4,5] работы, посвященные адсорбции газовых молекул на оксидных полупроводниках $\left(\mathrm{H} / \mathrm{ZnO}, \mathrm{O}_{2} / \mathrm{ZnO}\right.$ и $\left.\mathrm{O}_{2} / \mathrm{TiO}_{2}\right)$, доказавшие прямую пропорциональность между значениями $\Delta G(\Theta)$ и $\Delta \phi(\Theta)$, где $\Theta=N_{a} / N_{M L}-$ степень покрытия, $N_{a}\left(N_{M L}\right)$ - концентрация частиц в адслое (в монослое). Теория, связывающая изменения $\Delta G(\Theta)$ и $\Delta \phi(\Theta)$, была развита в $[6,7]$. В настоящей работе на основании этой теории мы проанализируем появившиеся недавно экспериментальные результаты по влиянию молекул газа на работу выхода и проводимость слоев наноструктурированных графита и графена.

\section{Теоретические соотношения}

В работах [6,7] было показано, что изменение концентрации носителей равно

$$
\Delta G(\Theta)=e \Delta n(\Theta) \mu(\Theta), \quad \Delta n(\Theta)=\Theta Z_{a}(\Theta) N_{M L} .
$$

С другой стороны, из теории адсорбции известно [1], что

$$
\Delta \phi(\Theta)=-\Phi \Theta Z_{a}(\Theta),
$$

где $\Phi=4 \pi e^{2} N_{M L} l, 2 l-$ толщина двойного электрического слоя, образованного заряженными адатомами и их изображением в подложке ( $l$ называют иногда длиной адсорбционной связи), $e$ - заряд позитрона. Таким образом, в предположении, что основную роль играет именно изменение концентрации носителей, а не подвижности, т. е. $|\Delta n| / n \gg|\Delta \mu| / \mu$, получаем следующее отношение $[1,6,7]$ :

$$
\eta \equiv\left|\frac{\Delta G(\Theta)}{\Delta \phi(\Theta)}\right|=\frac{\mu}{4 \pi e l} .
$$


Характерная особенность выражения (3) - отсутствие явной зависимости отношения $\eta$ от степени покрытия $\Theta$. Анализ данных по адсорбции молекул газа на полупроводниках показал, что выражение (3) вполне адекватно описывает экспериментальные результаты.

\section{Адсорбция на наноструктурированном графите}

В работах $[8,9]$ были проведены одновременные измерения $\Delta G$ и $\Delta \phi$, наводимые молекулами $\mathrm{NO}_{2}, \mathrm{NH}_{3}$, паров воды, ацетона и метанола, адсорбированными на многослойной пленке наноструктурированного графита (НГ), содержащей как кристаллические, так и аморфные области. При этом анализировалось отношение

$$
\xi=\frac{\Delta \phi}{\left(\Delta G / G_{0}\right)},
$$

где $G_{0}$ - проводимость графитовой пленки в отсутствие адсорбции. Анализ выявил прямую пропорциональность между изменениями $\Delta G$ и $\Delta \phi$, не зависящую от времени экспозиции в газе, т.е. от концентрации адчастиц. На этом основании было высказано предположение о том, что подвижность носителей при адсорбции практически не меняется, что подтверждает основной вывод теории $[6,7]$. При этом были получены следующие значения отношения $\xi$ (в единицах $\mathrm{meV} / \%):+75(\Delta \phi>0$, $\Delta G>0)$ для $\mathrm{NO}_{2},-45(\Delta \phi>0, \Delta G<0)$ для $\mathrm{NH}_{3}$, $+12(\Delta \phi<0, \Delta G<0)$ для $\mathrm{H}_{2} \mathrm{O},-110(\Delta \phi>0, \Delta G<0)$ для ацетона и $-13(\Delta \phi>0, \Delta G<0)$ для метанола [9]. Таким образом, исходя из знака $\Delta \phi$, следует, что все адсорбируемые молекулы, кроме $\mathrm{H}_{2} \mathrm{O}$, являются акцепторами, вызывающими увеличение работы выхода системы.

В соответствии с расчетами [10] при адсорбции на собственном графене молекулы $\mathrm{NO}_{2}$ являются акцепторами, $\mathrm{NH}_{3}$ - донорами, а $\mathrm{H}_{2} \mathrm{O}$ - донорами или акцепторами в зависимости от геометрии адсорбционного комплекса. По данным [11] при адсорбции на графене, допированном бором или азотом, молекулы $\mathrm{NO}_{2}$ и $\mathrm{NH}_{3}$ по-прежнему являются соответственно акцепторами и донорами. С другой стороны, акцепторы должны увеличивать (уменьшать) дырочную (электронную) проводимость пленки НГ [6,7]. Тогда, согласно [9], получается, что до адсорбции молекул $\mathrm{NO}_{2}$ и $\mathrm{H}_{2} \mathrm{O}$ пленка НГ обладала $p$-типом проводимости, тогда как до адсорбции молекул $\mathrm{NH}_{3}$, ацетона и метанола имела $n$-тип проводимости. Таким образом, для случая адсорбции $\mathrm{NH}_{3}$ в работе [9] имеется качественное противоречие.

Используя для $\Delta G$ и $\Delta \phi$ формулы (1) и (2), получим вместо (4) выражение

$$
\zeta=-4 \pi e^{2} l c_{0}
$$

где $c_{0}-$ поверхностная концентрация носителей в отсутствие адсорбции, равная $n_{0}\left(-p_{0}\right)$ для пленки НГ, обладающей соответственно $n(p)$-типом проводимости. $\mathrm{B}$ единицах $\mathrm{meV} / \%$ для $p$-типа проводимости имеем $|\xi| \approx 691 \cdot l c_{0}^{\prime}$, где $l$ измеряется в $\AA$ и $c_{0}^{\prime}=c_{0} /\left(3 \sqrt{3} a^{2} / 4\right)$ есть число носителей, приходящихся на один атом монослоя углерода в НГ $(a=1.42 \AA)$.

Расчеты [10] дают для адсорбции на собственном однолистном графене следующие значения длины десорбционных связей $l$ (в $\AA$ ): 3.6-3.9, 3.9-4.1, 3.5-4.1 для $\mathrm{NO}_{2}, \mathrm{NH}_{3}$ и $\mathrm{H}_{2} \mathrm{O}$ соответственно. Согласно [11], при допировании графена бором для адмолекул $\mathrm{NO}_{2}$ и $\mathrm{NH}_{3}$ имеем $l=1.67$ и $1.66 \AA$, тогда как в случае примеси азота $l=2.87$ и $2.86 \AA[12]$. В [12] для адмолекул $\mathrm{NO}_{2}, \mathrm{NH}_{3}$ и $\mathrm{H}_{2} \mathrm{O}$ на графене приводятся следующие значения $l$ (в $\AA$ ): 1.56, 3.37 и 3.73 при допировании бором; 3.03, 3.49 и 3.47 при допировании азотом. Несмотря на разброс результатов, ясно, что различием длин связи $l$ невозможно объяснить шестикратное отличие отношений $\xi$ для $\mathrm{NO}_{2}$ и $\mathrm{H}_{2} \mathrm{O}$ [9]. Поэтому, согласно (5), эти отличия значений $\xi$ диктуются разницей начальных концентраций носителей $c_{0}$. Используя формулу (5) и результаты работы [9], получим $\left|c_{0}^{\prime}\right| \approx 0.01-0.05$. Поэтому использование для нормировки изменения проводимости (выражение (4)) значения $G_{0}$ вызывает сомнения. Именно это обстоятельство препятствует пересчету полученных в [9] результатов по формулам (1)-(3).

\section{Адсорбция на графене}

Исследование адсорбционных свойств графена началось с работы [13], где изучалось влияние адсорбции молекул $\mathrm{NO}_{2}, \mathrm{H}_{2} \mathrm{O}$, йода (акцепторы) и $\mathrm{NH}_{3}, \mathrm{CO}$, этанола (доноры) на поверхностную проводимость механически отщепленного монослоя графена на окисленном кремнии и было показано, что $\Delta G \propto \Delta N$, а подвижность носителей $\left(\mu \approx 5000 \mathrm{~cm}^{2} / \mathrm{Vs}\right)$ не меняется. Таким образом, основной вывод теории [6,7] подтверждается.

В работе [14] исследовалась адсорбция молекул $\mathrm{NO}_{2}$ на слоях графена, сформированного на $\mathrm{Si}$ - и С-гранях $6 \mathrm{H}-\mathrm{SiC}$ (1-5 монослоев на Si-грани и 12-20 монослоев на С-грани). При этом знак изменения проводимости $\Delta G$

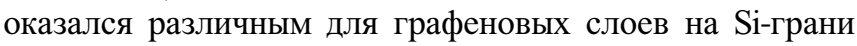
$(\Delta G<0)$ и С-грани $(\Delta G>0)$, что должно отвечать $n$ - и $p$-типам проводимостей этих граней. Аналогичное изменение знака наблюдалось для адсорбции $\mathrm{CO}_{2}, \mathrm{H}_{2} \mathrm{O}$, $\mathrm{NH}_{3}, \mathrm{O}_{2}, \mathrm{~N}_{2}$. Эти результаты, однако, находятся в противоречии с холловскими измерениями (см. подробнее [14]). Более того, нам вообще не известны работы, где бы отмечалось различие типов проводимости для Siи С-граней карбида кремния. В [14] также определялись значения $\Delta \phi$ при адсорбции $\mathrm{NO}_{2}$ и $\mathrm{NH}_{3}$ : в первом случае значения $\Delta \phi$ для обеих граней отрицательны, тогда как во втором положительны. Отсюда следует, согласно (2), что $\mathrm{NO}_{2}$ является донором, а $\mathrm{NH}_{3}$ акцептором, что противоречит всем предыдущим результатам по адсорбции на углеродных наноструктурах [8-13]. В связи с этим отметим, что в работе [15] те же знаки изменения 
работы выхода $\Delta \phi$ приводятся для адсорбции $\mathrm{NO}_{2}$ на $\mathrm{Si}-$ и С-гранях $6 \mathrm{H}-\mathrm{SiC}$ в отсутствие графеновых слоев. Тут, по нашему мнению, требуются дополнительные исследования.

В работе [16], где изучалось влияние адсорбции $\mathrm{NO}_{2}$ и $\mathrm{NH}_{3}$ на графеновые слои (3-4 монослоя) p-типа, помещенные на $\mathrm{SiO}_{2}$-подложку, при обработке экспериментальных данных используется независимое от концентрации адсорбата значение подвижности носителей $\mu=10.15 \mathrm{~cm}^{2} / \mathrm{Vs}$, которое представляется необъяснимо низким. Действительно, полагая $\Delta G \sim 10^{-3} \Omega^{-1}$, $\Delta \phi \sim 0.1 \mathrm{eV}$ (что по порядку величины соответствует данным [16]) и $l \sim 3 \AA$ [10-12], получим из соотношения (3) значение $\mu \sim 3000 \mathrm{~cm}^{2} / \mathrm{Vs}$, что вполне удовлетворительно согласуется с результатом работы [13]. Нам представляется, что значение $\mu=10.15 \mathrm{~cm}^{2} / \mathrm{Vs}$ в [16] возникает из-за некорректного пересчета экспериментальных данных.

Там же, в частности, в качестве любопытного по мнению авторов факта, приведено то обстоятельство, что при совпадении энергии адсорбционного уровня $\varepsilon_{a}$ молекулы $\mathrm{NO}_{2}$ с уровнем Ферми $E_{F}$ влияние адсорбированных молекул оказалось невозможным обнаружить [16]. Это, однако, неудивительно. Будем описывать плотность состояний двуэлектронного квазиуровня адатома контуром Лоренца

$$
\rho_{a}(\omega)=\frac{2}{\pi} \frac{\Gamma}{\left(\omega-\varepsilon_{a}\right)^{2}+\Gamma^{2}},
$$

где $\omega-$ энергия, Г- полуширина квазиуровня с энергией $\varepsilon_{a}$. Такая форма плотности состояний соответствует простейшему варианту модели Андерсона (приближение бесконечно широкой зоны, немагнитное решение $n_{a \uparrow}=n_{a \downarrow}=n_{a} / 2-$ см. подробнее в $\left.[1,17]\right)$. Считая температуру нулевой и интегрируя плотность состояний от $-\infty$ до $E_{F}$, получаем

$$
n_{a}=\frac{2}{\pi} \operatorname{arcctg}\left(\frac{\varepsilon_{a}-E_{F}}{\Gamma}\right),
$$

откуда при $\varepsilon_{a}=E_{F}$ имеем $n_{a}=1$ и $Z_{a}=0$. Следовательно, переход заряда отсутствует, что и делает невозможным детектирование адчастиц.

\section{Заключительные замечания}

Подводя итоги, следует прежде всего подчеркнуть, что, несмотря на все отмеченные нами противоречия в интерпретации экспериментальных данных в работах [8,9,14-16], бесспорным остается факт прямой пропорциональности изменений $\Delta G$ и $\Delta \phi$, вызванных адсорбцией, на чем и строится теория [6,7]. В работе [13] в связи с независимостью подвижности носителей от концентрации адчастиц предполагалось, что в случае однолистного графена заряженные примеси вообще не являются центрами рассеяния, а в качестве таковых выступают рипплы, возникающие вследствие спонтанной гофрировки листа. Еще ранее при изучении адсорбции газов на полупроводниковых оксидах было высказано предположение, что основной причиной рассеяния носителей является сама поверхность, а наличие на этой поверхности адчастиц представляется второстепенным обстоятельством [18]. Однако до настоящего времени вопрос о роли адчастиц и, шире, примесей в рассеянии носителей в графене остается открытым (см., например, [19-21]).

В предположении, что основным фактором изменений $\Delta G$ и $\Delta \phi$ является переход заряда между адсорбатом и адсорбентом, формулу (3) можно применять для порядковой оценки подвижности носителей $\mu$, взяв значение длины адсорбционной связи $l$ из расчетов типа [10-12] или приняв его, для простоты, равным постоянной решетки графена. Другой способ использования выражения (3) при известных значениях $\mu$ и $l-$ это расчет значений $\Delta G(\Theta)$ по экспериментально определенным значениям $\Delta \phi(\Theta)$ или, наоборот, расчет $\Delta \phi(\Theta)$ по данным для $\Delta G(\Theta)$.

\section{Список литературы}

[1] Давыдов С.Ю. Теория адсорбции: метод модельных гамильтонианов. СПб.: Изд-во СПбГЭТУ „ЛЭТИ“, 2013. $235 \mathrm{c}$.

[2] Göpel W. // Prog. Surf. Sci. 1985. Vol. 20. N 1. P. 9-103.

[3] Henrich V.E., Cox P.A. The Surface Science of Metal Oxides. Cambridge: University Press, 1994. 464 p.

[4] Давыдов С.Ю., Мошников В.А., Федотов А.А. // Письма в ЖТФ. 2004. Т. 30. Вып. 17. С. 39-44.

[5] Давыдов С.Ю., Мошников В.А., Федотов А.А. // ЖТФ. 2006. Т. 76. Вып. 1. С. 141-142.

[6] Аньчков Д.Г., Давыдов С.Ю., Трошин С.В. // Письма в ЖТФ. 2007. Т. 33. Вып. 18. С. 47-53.

[7] Аньчков Д.Г., Давыљов С.Ю., Трошин С.В. // Письма в ЖТФ. 2008. Т. 34. Вып. 18. С. 54-60.

[8] Qazi M., Vogt T., Koley G. // Appl. Phys. Lett. 2007. Vol. 91. P. 233101.

[9] Qazi M., Vogt T., Koley G. // Appl. Phys. Lett. 2008. Vol. 92. P. 103120.

[10] Leenaerts O., Partoens B., Peeters F.M. // Phys. Rev. B. 2008. Vol. 77. P. 125416

[11] Zhang Y.-H., Chen Y.-B., Zhou K.-G., Liu C.-H., Zeng J., Zhang H.-L., Peng Y. // Nanotechnology. 2009. Vol. 20. P. 185504.

[12] Dai J., Yuan J., Gianozzi P. // Appl. Phys. Lett. 2009. Vol. 95. P. 232105.

[13] Schedin F., Geim A.K., Morozov S.V., Hill E.W., Blake P., Katsnelson M.I., Novoselov K.S. // Nature Mater. 2007. Vol. 6. P. 652-655.

[14] Nomani Md.W.K., Shishir R., Qazi M., Diwan D., Shield V.B., Spencer M.G., Tompa G.S., Sbrockey N.M., Koley G. // Sens. and Actuat. B. 2010. Vol. 150. N 1. P. 301-307.

[15] Qazi M., Liu J., Chandrashekhar M.V.S., Koley G. // J. Appl. Phys. 2009. Vol. 106. P. 094901.

[16] Singh A.K., Uddin M.A., Tolson J.T., Maire-Afeli H., Sbrockey N., Tompa G.S., Spencer M.G., Vogt T., Sudarshan T.S., Koley G. // Appl. Phys. Lett. 2013. Vol. 102. P. 043101. 
[17] Давыдов С.Ю., Трошин С.В. // ФТТ. 2007. Т. 49. Вып. 8. C. $1508-1513$.

[18] Аньчков Д.Г., Давыдов С.Ю. // ФТТ. 2011. Т. 53. Вып. 4. C. $820-823$.

[19] Cheni J.-H., Jangi C., Adam S., Fuhrer M.S., Williams E.D., Ishigami M. // Nat. Phys. 2008. Vol. 4. N 5. P. 377-381.

[20] Wehling T.O., Katsnelson M.I., Lichtenstein A.I. // Chem. Phys. Lett. 2009. Vol. 476. N 4. P. 125-134.

[21] Yang Y., Brenner K., Murali R. // Carbon. 2012. Vol. 50. N 5. P. $1727-1733$. 